\title{
Selective detection of minor isotope lines in saturated absorption spectra by absorption filtering of major isotope lines
}

\section{AUTHOR(S):}

Onoda, Yugo; Sugiyama, Kazuhiko; Kitano, Masao

\section{CITATION:}

Onoda, Yugo ... [et al]. Selective detection of minor isotope lines in saturated absorption spectra by absorption filtering of major isotope lines. Optical Review 2011, 18(4): 365-366

\section{ISSUE DATE:}

2011-07

URL:

http://hdl.handle.net/2433/178701

\section{RIGHT:}

(C) 2011 Japan Society of Applied Physics; This is not the published version. Please cite only the published version.; この論文は出版社版で ありません。引用の際には出版社版をご確認ご利用ください。 


\title{
Selective Detection of Minor Isotope Lines in Saturated Absorption Spectra by Absorption Filtering of Major Isotope Lines
}

\author{
Yugo Onoda, ${ }^{1, *}$ Kazuhiko Sugiyama, ${ }^{1,2}$ and Masao Kitano ${ }^{1,2}$ \\ ${ }^{1}$ Graduate School of Electronic Science and Engineering, Kyoto University, Katsura, Nishikyo, Kyoto \\ 615-8510, Japan
}

${ }^{2}$ Core Research for Evolutional Science and Technology, Japan Science and Technology Corporation, 4-1-8 Honcho, Kawaguchi, Saitama, Japan

We demonstrate a simple method that can be used to detect minor isotope lines in a saturated absorption spectrum by the absorption filtering of major isotope lines. We investigate this method for use in the spectroscopy of the ${ }^{1} \mathrm{~S}_{0}-{ }^{1} \mathrm{P}_{1}$ transition in $\mathrm{Yb}$ at $399 \mathrm{~nm}$ by controlling the density of $\mathrm{Yb}$ atoms by varying the discharge current of a hollow cathode lamp. The performance of an extended-cavity laser diode using a high-power ultraviolet diode chip is also analyzed.

Keywords: saturated absorption spectroscopy, ytterbium, minor isotope, ultraviolet laser diode, isotope shift

\footnotetext{
${ }^{*}$ E-mail address: onoda@giga.kuee.kyoto-u.ac.jp
} 
OPTICAL REVIEW

Short Note

Saturated absorption (SA) is one of the techniques used in high-resolution spectroscopy and is applied to the detection of the transitions in atoms and molecules used to obtain reference frequencies. Even after employing SA, isotope shifts and hyperfine structures are sometimes incompletely resolved, and these unresolved lines contain signals of target isotopes required for subsequent investigations.

In this note, we describe a simple method that can be used in SA spectroscopy for the selective detection of minor isotope atoms. We assume that the intensity of a pump beam is greater than the saturation intensity at the entrance of the sample of atoms. As we increase the density of the atoms, a significant decrease in pump beam intensity caused by linear absorption is observed, and finally, the pump beam intensity decreases below the saturation intensity before the pump beam reaches the end of the sample. Under this condition, the probe beam, which enters the sample from the opposite side, is absorbed until it reaches the saturated part of the sample, i.e., we detect the SA signal using a probe beam with a lower intensity. On the other hand, the number of atoms that contribute to the SA signal does not increase as we further increase the density of atoms. The number of atoms that contribute to the SA signal increases at the entrance of the sample. However, the depth at which the pump beam intensity decreases below the saturation intensity is inversely proportional to the density of atoms. Therefore, when the depth at which the SA signal is generated is smaller than the sample length, the SA signal grows smaller as we further increase the density of atoms because the number of atoms that contribute to the SA signal, which is detected using the weakened probe beam by linear absorption, remains approximately constant. As we increase the density of atoms, the above phenomenon is first observed in isotopes that are 
highly abundant and then in isotopes that are less abundant. Therefore, we can selectively detect the SA signal of minor isotopes by adjusting appropriately the density of atoms. We demonstrate this in a commercially available hollow cathode lamp (HCL) by using ${ }^{1} \mathrm{~S}_{0}-{ }^{1} \mathrm{P}_{1}$ at $399 \mathrm{~nm}$ in $\mathrm{Yb}$ atoms. The HCL is conveniently used to obtain the reference frequencies ${ }^{1)}$ and the density of atoms is controlled by varying the discharge current of the HCL. This transition is used for the laser cooling of $\mathrm{Yb}^{2)}$ and the loading of $\mathrm{Yb}^{+}$in an ion trap through photoionization..$^{3)}$

The experimental setup is shown in Fig. 1. We construct an extended-cavity laser diode (ECLD) with a high-power ultraviolet diode chip having a maximum output power of $100 \mathrm{~mW}$ (Nichia, $8 \mathrm{C} 18 \mathrm{KS})$. A diffraction grating with 2400 grooves $/ \mathrm{mm}$ and a blaze wavelength of $250 \mathrm{~nm}$ is placed in a Littrow configuration. The first-order diffracted beam whose power is $25 \%$ of the incident power is fed back to the chip, and the zeroth-order reflection whose power is $64 \%$ of the incident power is produced as the output. The maximum power with which we achieve a single-frequency oscillation increases as the cavity length of the ECLD is reduced, as shown in Fig. 2. At the smallest cavity length of $14 \mathrm{~mm}$, we obtain a tuning range with a single-frequency oscillation of $2 \mathrm{~nm}$ and with a linewidth below 2 and $20 \mathrm{MHz}$ for observation times of 1 and $10 \mathrm{~ms}$, respectively. The linewidth is measured using the beat signals at the second-harmonic frequency of a titanium sapphire laser.

The laser beam from the ECLD is split into a strong pump beam and a weak probe beam. The two beams counterpropagate and overlap in an HCL (Hamamatsu Photonics, L2783-70ANEYB). The HCL contains a carrier gas comprising a mixture of Ar and Ne gases, which is injected into the HCL at pressures of 270 and $400 \mathrm{~Pa}$. The powers of the pump and probe beams are 360 
and $30 \mu \mathrm{W}$, respectively, at the window of the HCL. The scanning speed of the laser frequency is $15 \mathrm{MHz} / \mathrm{s}$. To eliminate the component of linear absorption, the pump beam is chopped and the probe beam is detected at the chopping frequency by using a lock-in amplifier. The typical chopping frequency is $1170 \mathrm{~Hz}$.

The SA spectra are shown in Fig. 3 as a function of the discharge current of the HCL. The lines of the isotopes and the hyperfine components are assigned by using the frequency intervals reported in the literature. ${ }^{4)}$ The natural abundance values of the Yb isotopes 176, 174, 173, $172,171,170$, and 168 are $13,32,16,22,14,3$, and $0.1 \%$, respectively. As the discharge current is increased, we first observe that all the lines grow larger in proportion to the increase in the density of $\mathrm{Yb}$ atoms. Above a discharge current of $2 \mathrm{~mA}$, the signals of the major isotopes 172 and 174 grow smaller, whereas the signal of the minor isotope 171 becomes more distinct, as discussed above. Because the lines of the two major isotopes are located around the center of the entire absorption structure, the lines at the edge of the structure for the isotopes 176,171 , 170, and 168 remain even at a high discharge current of more than $5 \mathrm{~mA}$. At a high current of more than $5 \mathrm{~mA}$, the probe beam is absorbed by the lines of the isotopes 176,171 , and 170 , and finally, we observe only the signal of the isotope 168, which is the rarest isotope. We can set the optimum discharge current for each isotope to detect the SA signal with the highest resolution. For example, for the isotope 171, which is used as an optical frequency standard, 5,6 ) the discharge current of $2.0-2.8 \mathrm{~mA}$ gives us the highest resolution. This method is used in the case of other atoms to selectively detect the lines of their minor isotopes. 
OPTICAL REVIEW

Short Note

\section{Acknowledgments}

The authors wish to thank Yoichi Kawakami of Kyoto University and Nichia Corporation for providing the ultraviolet laser diode. This work was partly supported by a Grant-in-Aid for Scientific Research (No. 20.6222), by the Global Center of Excellence program from the Japan Society for the Promotion of Science (JSPS), and by a Grant-in-Aid for Scientific Research on Innovative Areas "Extreme quantum world opened up by atoms" (No. 21104006) from the Ministry of Education, Culture, Sports, Science and Technology. 


\section{References}

1) T. Kohno, M. Yasuda, H. Inaba, and F.-L. Hong: Jpn. J. Appl. Phys. 47 (2008) 8856.

2) K. Komori, Y. Takasu, M. Kumakura, Y. Takahashi, and T. Yabuzaki: Jpn. J. Appl. Phys. $42(2003) 5059$.

3) C. Balzer, A. Braun, T. Hannemann, C. Paape, M. Ettler, W. Neuhauser, and C. Wunderlich: Phys. Rev. A 73 (2006) 041407.

4) D. Das, S. Barthwal, A. Banerjee, and V. Natarajan: Phys. Rev. A 72 (2005) 032506.

5) T. Kohno, M. Yasuda, K. Hosaka, H. Inaba, Y. Nakajima, and F.-L. Hong: Appl. Phys. Express 2 (2009) 072501.

6) T. Schneider, E. Peik, and C. Tamm: Phys. Rev. Lett. 94 (2005) 230801. 
Fig. 1 caption

Experimental setup of SA spectroscopy. PBS, polarizing beam splitter; PD, photodiode; ISR, isolator with isolation $>40 \mathrm{~dB}$.

Fig. 2 caption

Maximum output power achieved with single-frequency oscillation in ECLD with ultraviolet diode chip as a function of extended-cavity length.

Fig. 3 caption

Saturated absorption signals of ${ }^{1} \mathrm{~S}_{0}-{ }^{1} \mathrm{P}_{1}$ transition in $\mathrm{Yb}$ as a function of discharge current of HCL. (a) CO, crossover resonance. S1, S2, and S3 are transitions of 173(5/2), 173(3/2), and 173(7/2), respectively. ${ }^{4)}$ The number in parentheses represents the total angular momentum of the upper state of the transitions. (b) Dependence of SA signal height on discharge current for three isotope lines shown in the figure. We plot the peak height of the detected signals as the signal height for each line without the deconvolution of the components. 


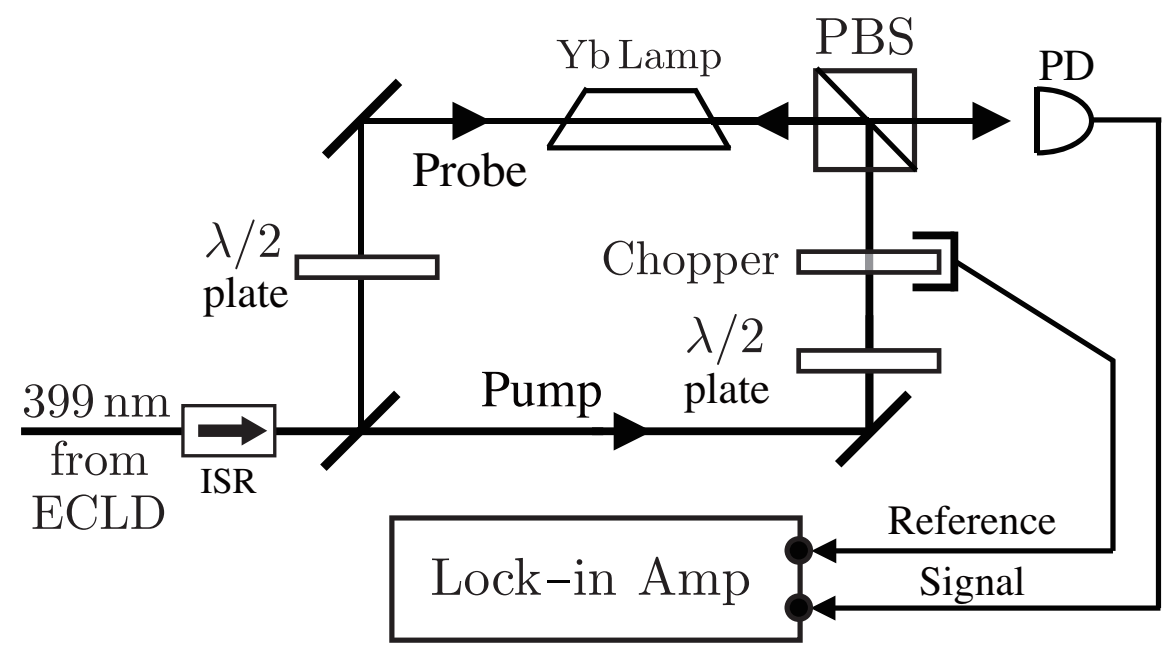

Fig. 1 .

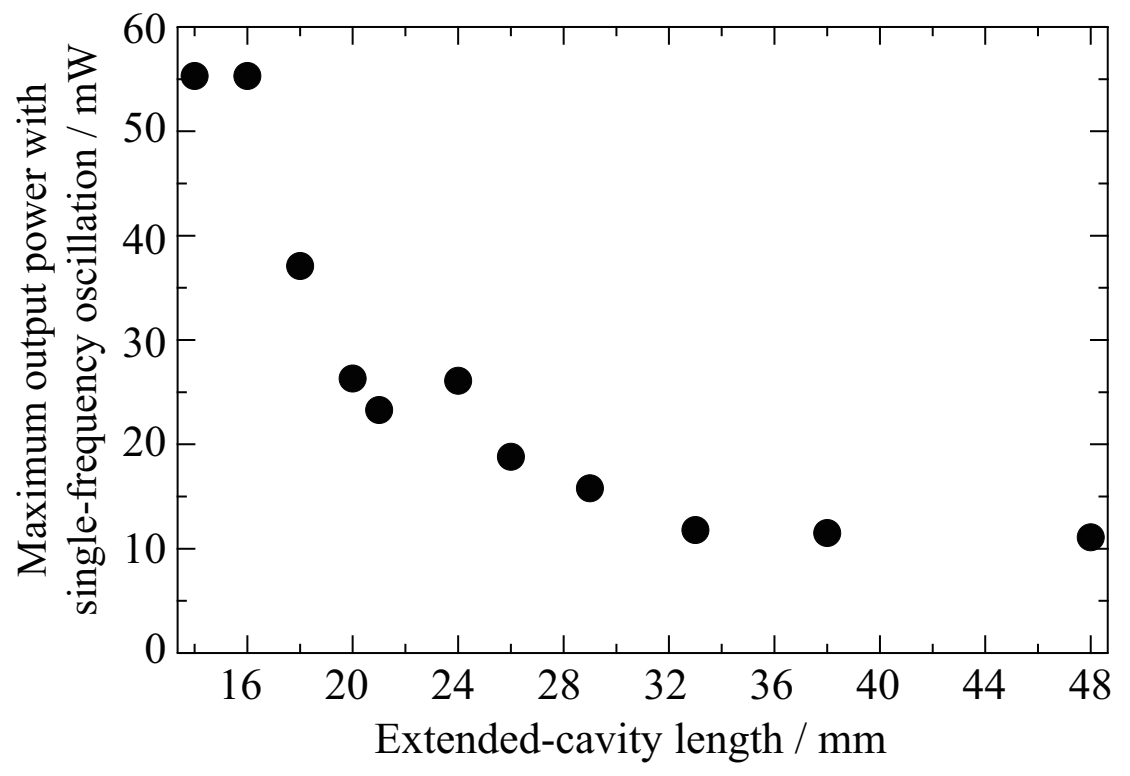

Fig. 2. 

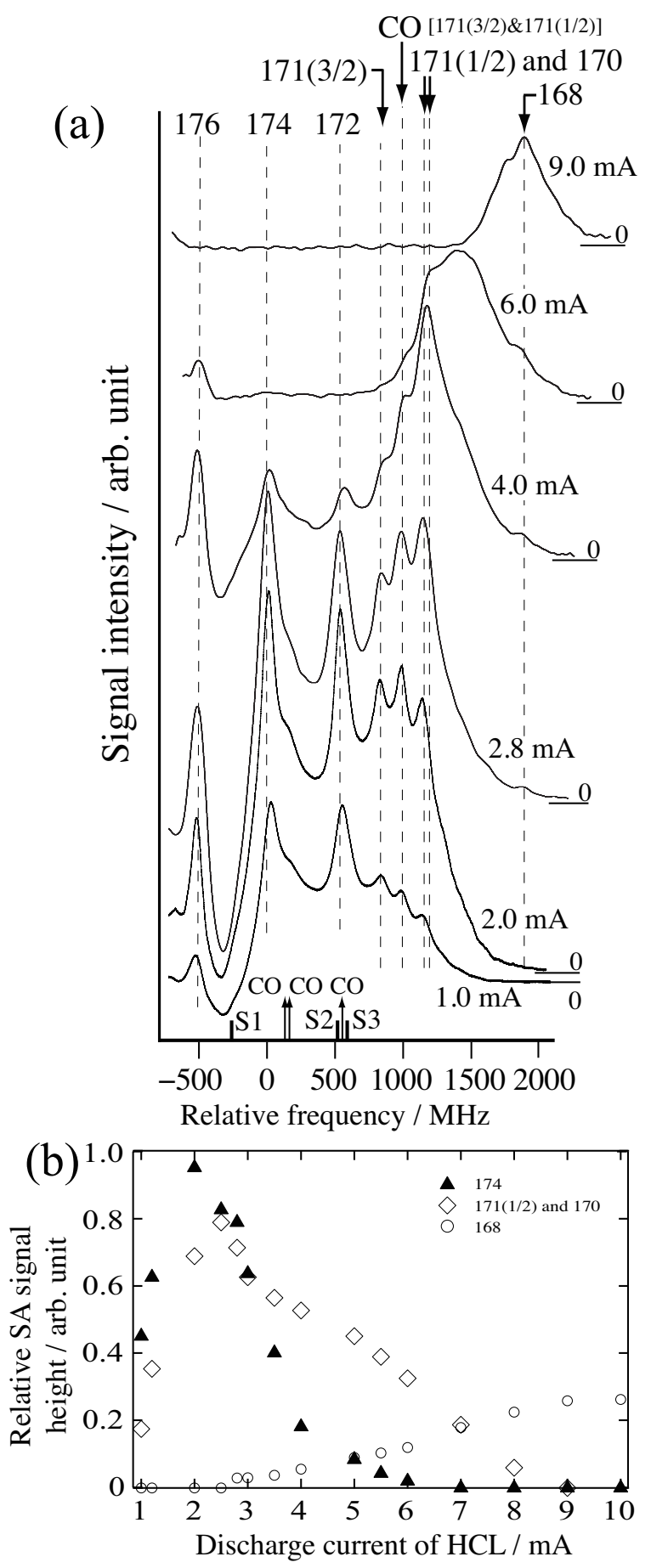

Fig. 3. 